\title{
Integrating prison health care into public health care: the global view
}

$\mathrm{T}$ welve years after Nova Scotia integrated the health care offered in its provincial prisons into its public health system, the province's Chief Public Health Officer, Dr. Robert Strang, says the benefits are obvious.

"There are so many public health problems in the correctional facilities, especially infectious diseases," he notes. "So it absolutely makes sense to have health services in them delivered by people who understand public health care and will promote linkages with the public system."

The difficulty of recruiting professional health staff for work in prisons and ensuring the quality of health

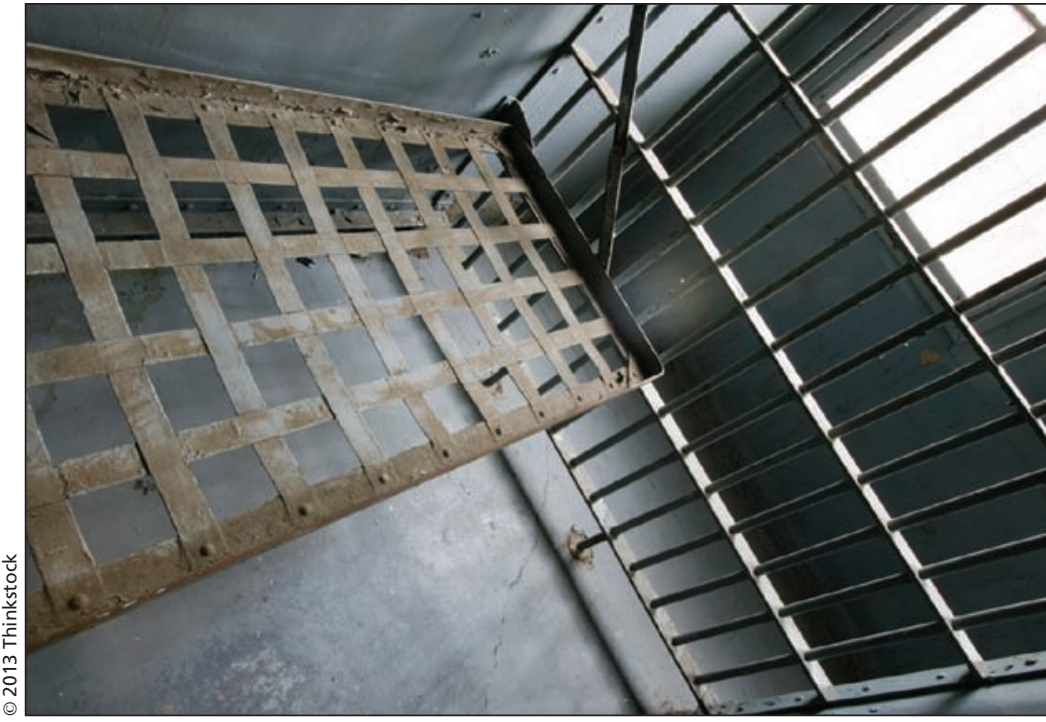

Improved public health, better recruitment of professional health staff and enhanced quality control are just a few of the advantages of a public system. she explains. "We did it in order to promote equity in health care in the prisons. Prisoners now receive their care from NHS - as does the general popu- hands of public health officials echoes that of most governments worldwide.

But the arguments in favour of doing so are overwhelming, he insists.

For starters, he explains, integrating prison health care into public health dramatically expands the pool of health care resources and expertise available in prisons. It also substantially increases transparency.

And it eliminates the problem of "dual loyalty" in which physicians and health staff working for prison systems find that their duty to serve patients may clash with their duty to serve prison officials. "If you don't give physicians in prisons complete independence, you damage the relationship lation. The transfer upholds European and international standards for the health care of prisoners.'

The World Health Organization (WHO) began promoting the integration of prison and public health care in 2003, when it issued the Moscow Declaration, which asserts that prison health and public health should "bear equal responsibility for health in prisons" in order to achieve improved prison heath standards and infectious disease control. "No country can afford to ignore widespread precursors of disease in prisons such as overcrowding, inadequate nutrition and unsatisfactory conditions," the declaration warned (www.euro.who.int/__data /assets/pdf_file/0007/98971/E94242.pdf).

In Copenhagen, Denmark, Stefan Enggist, the WHO's point person on prison health, says the Canadian government's refusal to even publicly discuss putting prison health care into the between doctors and patients and you damage public health,' Enggist explains.

But sensible as the notion of integrating prison health care into public health may be, prison administrators have been slow to accept it, notes Dr. Michael Levy, clinical director for Justice Health Services for the Australian Capital Territory in Canberra. Since 1978, he explains, health care has been transferred out of the hands of correctional officials in all but one of Australia's eight provinces.

'It doesn't happen overnight that a government changes over from a traditional paramilitary system," Levy warns. "There are countless imperatives for prison governors to take public health seriously, but they are also very effective at hiding the realities of health conditions in their facilities." — Paul Christopher Webster, Toronto, Ont. transferred health care in Scottish prisons to the National Health Service [NHS], and so far it's been a success."
CMAJ 2013. DOI:10.1503/cmaj.109-4435 